\title{
An Absolute Measurement of the Cosmic Microwave Background Radiation Temperature at $10.7 \mathrm{GHz}$
}

\author{
S. T. Staggs, N. C. Jarosik, ${ }^{1}$ S. S. Meyer, D. T. Wilkinson ${ }^{1}$ \\ Enrico Fermi Institute \\ University of Chicago, Chicago, IL 60637.
}

Received _ ; accepted _

\footnotetext{
${ }^{1}$ Joseph Henry Laboratories and Physics Department, Princeton University, Princeton, NJ 08544
} 


\begin{abstract}
A balloon-borne experiment has measured the absolute temperature of the cosmic microwave background radiation (CMBR) at $10.7 \mathrm{GHz}$ to be $T_{\mathrm{CMBR}}=2.730 \pm 0.014 \mathrm{~K}$. The error is the quadratic sum of several systematic errors, with statistical error of less than $0.1 \mathrm{mK}$. The instrument comprises a cooled corrugated horn antenna coupled to a total-power radiometer. A cryogenic mechanical waveguide switch alternately connects the radiometer to the horn and to an internal reference load. The small measured temperature difference $(\lesssim 20 \mathrm{mK})$ between the sky signal and the reference load in conjunction with the use of a cold front end keeps systematic instrumental corrections small. Atmospheric and window emission are minimized by flying the instrument at $24 \mathrm{~km}$ altitude. A large outer ground screen and smaller inner screen shield the instrument from stray radiation from the ground and the balloon. In-flight tests constrain the magnitude of ground radiation contamination, and low level interference is monitored through observations in several narrow frequency bands.
\end{abstract}

Subject headings: cosmology:cosmic background radiation — cosmology: observations 


\section{INTRODUCTION}

The residual radiation from the early universe retains a nearly blackbody spectrum today, allowing for its interpretation as a primeval remnant. While the present spatial anisotropy of the cosmic microwave background radiation (CMBR) maps out the positions of small inhomogeneities in the early universe at the epoch of decoupling, spectral distortions in the CMBR carry an imprint of the energetic history of the early universe. Processes releasing energy at redshift $z<10^{7}$ (see Wright et al. 1994 for examples) can cause distortions in the CMBR spectrum, either by directly altering the photon occupation number or by heating the matter which may then couple to the radiation. Several frequency-dependent processes, including nonradiative and radiative Compton scattering and free-free emission, slowly relax the radiation back toward a blackbody spectrum; thus the frequency signature of any distortion provides clues to both the nature and epoch of its origin. Smoot \& Scott 1996 give a review of distortions and recent experimental results. The parameter $Y_{f f}$ describes distortions at long wavelengths arising from free-free emission during an epoch of re-ionization, while $y$ parameterizes Compton distortions (most noticeable at short wavelengths) which have not yet undergone much relaxation. The chemical potential $\mu$ parameterizes distortions in which the CMBR spectrum has relaxed to kinetic equilibrium, but lacks the number of photons needed for a blackbody spectrum.

The FIRAS instrument on the COBE satellite measured the CMBR spectrum between $60 \mathrm{GHz}$ and $630 \mathrm{GHz}$ very precisely (Fixsen et al. 1996), determining $T_{\mathrm{CMBR}}$ to be $2.728 \pm .004 \mathrm{~K}(95 \% \mathrm{CL})$ and constraining $y<1.5 \times 10^{-5}$ and $\mu<9 \times 10^{-5}$. At longer wavelengths, existing measurements are substantially less accurate. All extant data taken together constrain $Y_{f f}<1.9 \times 10^{-5}$ (Smoot \& Scott 1996). Most progress on measurements of or limits on $\mu$ and $Y_{f f}$ will now come from improved longer wavelength data. This paper presents a recent accurate measurement of $T_{\mathrm{CMBR}}$ at $10.7 \mathrm{GHz}$. 


\section{EXPERIMENT DESIGN}

The balloon-borne apparatus (Figure 1$)$ features a cold $(\approx 5 \mathrm{~K})$ corrugated horn antenna with a $15^{\circ}$ FWHM beam coupled through a cold waveguide switch to a total power radiometer. The switch alternately connects the radiometer to the antenna signal and to an internal temperature-regulated reference load, consisting of iron-loaded epoxy absorber thermally sunk inside a $2.8 \mathrm{~kg}$ copper waveguide block. The load's measured reflection is $<-30 \mathrm{~dB}$. The switch period is 85 seconds. The horn antenna views the sky through a thin $\left(0.001^{\prime \prime}\right)$ polypropylene window which separates the dewar vacuum from the ambient pressure at float $(\approx 20$ torr $)$. [t The input of the radiometer incorporates a cryogenic circulator and a second temperature-regulated load to mitigate errors associated with small differences in the reflection coefficients of the horn and reference load. The temperatures of the two loads are measured with calibrated germanium resistance thermometers (Lake Shore model GR-200A-2500) and read out with precision AC bridges. During the flight these temperatures were stable to better than $0.1 \mathrm{mK}$ over time scales of several minutes. Additional temperature regulators maintained the switch and the circulator body within $\approx 100 \mathrm{mK}$ of the sky signal during flight.

The signal is amplified in a cryogenic 9-12 GHz HEMT amplifier supplied by the NRAO. Subsequently, the signal bandpass is limited to $10-11 \mathrm{GHz}$ in a fixed roomtemperature filter. Two fixed filters and two tunable filters divide the signal into four frequency bands. One linear polarization is detected. During the flight, one of the tunable filters (with $50 \mathrm{MHz}$ bandwidth) was continuously stepped through the $1 \mathrm{GHz}$ bandpass to monitor possible radiofrequency interference; none was seen. The system noise temperature was $12 \mathrm{~K}$. This paper reports the results from the two fixed filters, with bandpasses

\footnotetext{
${ }^{2}$ The window is too thin to withstand atmospheric pressure; a metal plate seals off the dewar vacuum on the ground and is pulled out of the way in flight.
} 
$10.625 \pm .025 \mathrm{GHz}$ (Channel 2) and 10.69 $\pm .01 \mathrm{GHz}$ (Channel 3).

The gondola hangs $\approx 1100^{\prime}$ below the balloon, which has a diameter of $\approx 175^{\prime}$ at float. The gondola rotated about once a minute for much of the flight, so that the instrument observed a broad swath of the sky. Most of the data were taken with the beam center at a zenith angle of $40^{\circ}$. The antenna beam has very low, symmetric sidelobes, and is shielded from ambient temperature emission from the earth and the balloon by an inner ground screen attached to the dewar; with this screen in place, the measured sidelobe response is below $-55 \mathrm{~dB}$ for all angles greater than $50^{\circ}$ from the beam axis. A fixed outer ground screen provides additional rejection of ground radiation.

\section{DATA}

The instrument was launched from Fort Sumner, NM, on December 10, 1995. The radio command uplink became intermittent a few minutes after the package reached float altitude, severely restricting the program of measurements. The data divide into three segments chronologically, according to the zenith angles of observation: the first $40^{\circ}$ tip, the $60^{\circ}$ tip, and the second $40^{\circ}$ tip. Details of these data segments are given in Table 1 . In

the latter two data segments, the instrument observed the moon, providing a crosscheck of the pointing, beamsize, and gain. Continuous in-flight gain calibration was accomplished by alternating the temperature of the reference load, $T_{r e f}$, between $2.749 \mathrm{~K}$ and $4.091 \mathrm{~K}$ at twice the switch period. The temperature difference between the colder of the two setpoints and the sky was $\lesssim 20 \mathrm{mK}$, so that the effect of gain errors in determining the sky temperature is negligible. Table 1 includes raw numbers for the temperature of the sky signal, with no corrections for any systematic or instrument effects. 


\section{SYSTEMATIC EFFECTS}

Five sources of extraneous signal are described here with estimates of the errors in removing those sources. The results are summarized in Table 2. The commanding difficulties precluded planned in-flight measurements of several of these systematic effects, so that calculations are used instead. However, the experiment design keeps the size of all the corrections small enough to allow for a precise determination of $T_{\mathrm{CMBR}}$.

\subsection{Radiometer Offset}

The radiometer operation has been studied with multiple separate cooldown tests in which the sky horn is removed from the dewar and replaced with a second thermally regulated waveguide load, identical in construction to the reference load. In particular, two

such calibration tests took place in Fort Sumner just before the launch. Flight conditions are approximated during these tests by pumping on the helium bath.

If the radiometer were ideal, when the measured temperatures of the two waveguide loads were equal, no differential signal would be observed from the radiometer. This radiometer instead exhibits offsets of $T_{\text {off }}=7 \mathrm{mK}$ and $10 \mathrm{mK}$ in channels 2 and 3 respectively. Four independent measurements of these offsets were made in four separate cooldowns which agree to the relative accuracy of the measurements, about $2 \mathrm{mK}$. These measured offsets are assumed to apply during the flight. However, since their origin is not yet certain, errors equal to the magnitudes of the offsets are assigned to their removal. 


\subsection{Emission and Reflection from Waveguide/Optics}

The emission expected from the corrugated horn at $5 \mathrm{~K}$ is calculated according to the methods described in Clarricoats, Olver \& Chong (1975). The horn was fabricated as a single piece to eliminate the excess emission associated with joints. The effect of the short waveguide transition coupling the horn to the waveguide switch is included in the

calculation, yielding $T_{\text {horn }}=7_{-3}^{+7} \mathrm{mK}$. The errors are conservative estimates. The measured reflection coefficent of the horn is $<-28.5 \mathrm{~dB}$ and that of the window is $<-45 \mathrm{~dB}$. Since the in-flight circulator load temperature was within $100 \mathrm{mK}$ of the sky and reference load temperatures, differential reflection effects are negligible. The expected window emission, $1 \pm 1 \mathrm{mK}$, is interpolated from measurements at other frequencies (Chantry et al. 1971).

The ambient-temperature $(211 \pm 2 \mathrm{~K})$ inner ground screen (IGS), which is fixed to the dewar, is near enough to the beam to contribute $T_{I G S}=3 \pm 3 \mathrm{mK}$ to the signal. The magnitude of this effect was determined after the flight by lining the inner surface of the inner ground screen with microwave absorber and measuring this lining's contribution to the sky signal. These measurements were made by alternately observing the sky with and without the absorber lining while operating the radiometer at ambient temperature to obviate the need for a thick emissive window on the dewar. These results were scaled to account for the much lower emissivity of the aluminum relative to the microwave absorber, and the reduced effective emissivity of the absorber at low incidence angles.

\subsection{Ground and Balloon Emission}

When the beam's zenith angle changed from $40^{\circ}$ to $60^{\circ}$ during the flight, the observed sky temperature increased by $\Delta T_{t i p} \approx 10 \mathrm{mK}$. (See Table 1.) Ground calibration tests indicate that the radiometer offset is unaffected by tipping, allowing attribution of $\Delta T_{\text {tip }}$ 
to an increase in the total radiation entering the antenna. The associated atmospheric increase is expected to be $1.6 \pm 1.6 \mathrm{mK}$ (see Section 4.4), while the change in astrophysical foreground emissions is $-0.4 \pm 1.4 \mathrm{mK}$ (see Section 4.5). The remaining increase in sky temperature, $\Delta T_{\text {gnd }} \approx 9 \mathrm{mK}$, is used to constrain the magnitude of the ground radiation, $T_{\text {gnd }}$, entering the antenna during the $40^{\circ}$ tip. The largest contribution to $T_{\text {gnd }}$ comes from ground radiation diffracting over the front edge of the ground screen. Measured beam patterns of the horn antenna with the IGS in place indicate that the received ground pickup from over the front edge should increase by a factor of $\gtrsim 100$ upon tipping from $40^{\circ}$ to $60^{\circ}$. The small value of $\Delta T_{\text {gnd }}$ therefore implies that $T_{g n d}$ is less than $1 \mathrm{mK}$. Since calculations of diffracted ground signals are notoriously difficult, these estimates have been checked with further measurements on the ground. Prior to flight, direct measurements were made of the system beam map with both ground shields in place. The entire gondola was tipped relative to a distance source, with the dewar fixed at several different angles relative to the gondola. These spot measurements agreed with predictions. Nonetheless, a conservative limit of $T_{\text {gnd }}<5 \mathrm{mK}$ is adopted. In principle radiation from the ground might reflect off

the balloon and into the horn antenna, but since the balloon fills only 0.02 sr and has small reflectivity, this effect is negligible.

\subsection{Atmospheric Emission}

Extrapolation of the model described by Danese \& Partridge (1989) to the flight altitude of $25 \mathrm{~km}$, using the standard reference atmosphere for January midlatitudes $\left(30^{\circ} \mathrm{N}\right.$, Dursa 1985) yields $T_{a t m}=3 \mathrm{mK}$ at $40^{\circ}$ zenith angle. The average altitude dropped by $1.5 \mathrm{~km}$ from the first $40^{\circ}$ tip to the second, providing an opportunity to test the model. The model predicts a $2 \mathrm{mK}$ increase due to this drop; the measured increase is $2 \pm 1 \mathrm{mK}$. 


\subsection{Foreground Emission}

The principal celestial foreground emitters are the moon and the Galaxy; the data are also corrected for the dipole of the CMBR, as observed by COBE (Fixsen et al. 1996). The dipole effect varies from $-3 \mathrm{mK}$ to $+2 \mathrm{mK}$ across the region of sky observed. The Galactic signal is extrapolated from the continuum data at $408 \mathrm{MHz}$ (Haslam, Quigley, \& Salter 1970; Haslam et al. 1974; Haslam et al. 1981) using a spectral index of $2.8 \pm 0.1$. The Galactic signal varies from $2 \mathrm{mK}$ to $7 \mathrm{mK}$ in these data. The Galactic signal is removed after binning the data into sky coordinates.

After the position-dependent sum of the dipole and Galactic signals is subtracted from

the data, the sky temperature data for the second two data segments are plotted versus the angle subtended from the beam center to the moon's position. A Gaussian beam profile is then fit to these data, reconfirming the measured beamwidth of $15^{\circ} \mathrm{FWHM}$ and implying an emission temperature for the moon of $240 \mathrm{~K}$ with the moon about three-quarters full. This fit is used to correct the data for the moon's signal. All data for which the moon signal is in excess of $10 \mathrm{mK}$ ( $7 \%$ of the data) are excluded from the determination of $T_{\mathrm{CMBR}}$.

The average sum of these three foregrounds over the two $40^{\circ}$ tips is $T_{\text {fgnd }}=2 \pm 2 \mathrm{mK}$.

\section{RESULTS AND CONCLUSIONS}

The data and their systematic errors are summarized in Table 2. The final result, averaged over the two bands, is a measurement of $T_{\mathrm{CMBR}}=2.730 \pm 0.014 \mathrm{~K}$ at $10.7 \mathrm{GHz}$, a measure accurate to half a percent, made without recourse to space missions. It is in agreement with a previous measurement of $T_{\mathrm{CMBR}}=2.61 \pm 0.06 \mathrm{~K}$ at $10 \mathrm{GHz}$ (Smoot et al. 1987), and with the extremely precise FIRAS measurement of $T_{\mathrm{CMBR}}=2.728 \pm 0.004 \mathrm{~K}$ (Fixsen et al. 1996). Several more measurements of $T_{\mathrm{CMBR}}$ with accuracies comparable to 
the results described here will significantly tighten the constraints on $\mu$ and $Y_{f f}$.

We thank Skip Johnson, Ted Griffiths, Bill Dix, Laszlo Varga, Al Dietrich and Glenn Atkinson for help in mechanical design and construction. We are grateful to Richard Bradley and the NRAO for supplying the HEMT amplifer. We express our gratitude to the NSBF staff in Palestine and Fort Sumner for the excellent support before, during and after the launch of the balloon, particularly Mark Cobble.

This work was supported by NSF grant \# PHY89-21378. Additional support was provided by NASA through Hubble Fellowship grant \#HF-01063.01-94A awarded by STScI, which is operated by the Association of Universities for Research in Astronomy, Inc., for NASA under contract NAS 5-26555. 


\section{REFERENCES}

Chantry, G. W., Fleming, J. W., Pardoe, G. W. F., Reddish, W., \& Willis, H. A. 1971, Infrared Physics, 11, 109

Clarricoats, P. J. B., Olver, A. D., \& Chong, S. L., 1975 Proc. IEE, 122, 11, 1173

Danese, L., \& Partridge, R. B. 1989, ApJ, 342, 604; See also Crane, R. K., in Astrophysics, Vol. 12, Methods of Experimental Physics, ed. Meeks, M. L., (New York: Academic Press), 136; and Liebe, Hans J. 1985, Radio Science, 20, 5, 1069

Fixsen, D. J., Cheng, E. S., Gales, J. M., Mather, J. C., Shafer, R. A., \& Wright, E. L. 1996, ApJ, in press

Haslam, C. G. T., Quigley, M. J. S., \& Salter, C. J. 1970, MNRAS, 147, 405

Haslam, C. G. T., Salter, C. J., Stoffel, H., \& Wilson, W. E. 1974, A\&AS, 13, 369

Haslam, C. G. T., Klein, U., Salter, C. J., Stoffel, H., Wilson, W. E., Cleary, M. N., Cooke, D. J., \& Thomasson, P. 1981, A\&A, 100, 209

Jursa, A. S. (ed.) 1985, Handbook of Geophysics and the Space Environment, (Springfield, Va: Air Force Geophysical Laboratory), 14-1

Smoot, G. F., \& Scott, D. 1996, astro-ph/9603157

Smoot, G. F., Bensadoun, M., Bersanelli, M., De Amici, G., Kogut, A., Levin, S., \& Witebsky, C. 1987, ApJ, 317, L45

Wright, E. L., et al. 1994, ApJ, 420, 450 
Fig. 1. - Side view of the gondola with the beam's zenith angle at $40^{\circ}$, with an inset schematic of the radiometer. Windowscreen constitutes the outer ground screen, the sides of which make an angle of $30^{\circ}$ to the vertical. The inner ground screen is rolled aluminum. The wooden deck is covered with aluminum. The gondola is suspended from the balloon's flight train by the stainless steel cables depicted. Two of the radiometer channels are selected via tunable filters. Room-temperature isolators have been omitted for clarity. 
Table 1. DESCRIPTION OF THE THREE DATA SEGMENTS.

\begin{tabular}{ccccc}
\hline \hline Zenith Angle & $\begin{array}{c}\text { GMT Range } \\
(\mathrm{hrs})\end{array}$ & $\begin{array}{c}\text { Average Altitude } \\
(\mathrm{km})\end{array}$ & $\begin{array}{c}\text { Sky Signal } \\
\text { Ch 2 }(\mathrm{mK})\end{array}$ & $\begin{array}{c}\text { Sky Signal } \\
\text { Ch 3 }(\mathrm{mK})\end{array}$ \\
\hline \multirow{2}{*}{ First $40^{\circ}$} & $2.78-4.16$ & 25.3 & 2738 & 2735 \\
$60^{\circ}$ & $4.34-4.84$ & 28.6 & 2749 & 2746 \\
Second $40^{\circ}$ & $5.51-6.54$ & 23.8 & 2741 & 2739 \\
\hline
\end{tabular}

Note. - The gondola rotates $360^{\circ}$ in azimuth about once a minute. The signal levels shown for Channels 2 and 3 are the sums of the raw measured temperature differences between the sky and the reference load, with the reference load temperature $2749 \pm 4 \mathrm{mK}$. No corrections for any systematic effects have been applied. The statistical errors for each entry are $\lesssim 0.1 \mathrm{mK}$. 
Table 2. MEASURED SIGNALS AND SYSTEMATIC ERRORS

\begin{tabular}{lrr}
\hline \hline Parameter & $\begin{array}{c}\text { Channel } 2 \\
(\mathrm{mK})\end{array}$ & $\begin{array}{c}\text { Channel } 3 \\
(\mathrm{mK})\end{array}$ \\
\hline & $2749 \pm 4$ & $2749 \pm 4$ \\
$T_{\text {ref }}$ & $-10 \pm 1$ & $-12 \pm 1$ \\
$\Delta T_{\text {meas }}$ & $7 \pm 7$ & $10 \pm 10$ \\
$-T_{\text {off }}$ & $-7_{-7}^{+3}$ & $-7_{-7}^{+3}$ \\
$-T_{\text {horn }}$ & $-1 \pm 1$ & $-1 \pm 1$ \\
$-T_{\text {win }}$ & $-3 \pm 3$ & $-3 \pm 3$ \\
$-T_{I G S}$ & $>-5$ & $>-5$ \\
$-T_{\text {gnd }}$ & $-3 \pm 3$ & $-3 \pm 3$ \\
$-T_{\text {atm }}$ & $-2 \pm 2$ & $-2 \pm 2$ \\
$-T_{\text {fgnd }}$ & $2730_{-13}^{+10}$ & $2731_{-15}^{+12}$ \\
$T_{\mathrm{CMBR}}$ & & \\
\hline
\end{tabular}

Note. $-T_{\mathrm{CMBR}}$ in each channel is the sum of all the rows. The final error is obtained by summing the constituent errors in quadrature. $\Delta T_{\text {meas }}$ is the measured difference between the reference load and the sky signal for all the data at zenith angle $40^{\circ}$; the error includes statistical and gain effects. $T_{I G S}$ denotes emission from the inner ground screen. The foreground term includes effects from both the Galaxy (with an average value of $3 \mathrm{mK}$ ) and the CMBR dipole (which decreases the signal by about $1 \mathrm{mK}$ ). Channel 2 spans the range $10.68-$ $10.70 \mathrm{GHz}$, while Channel 3 ranges from $10.6 \mathrm{GHz}$ to $10.65 \mathrm{GHz}$. 


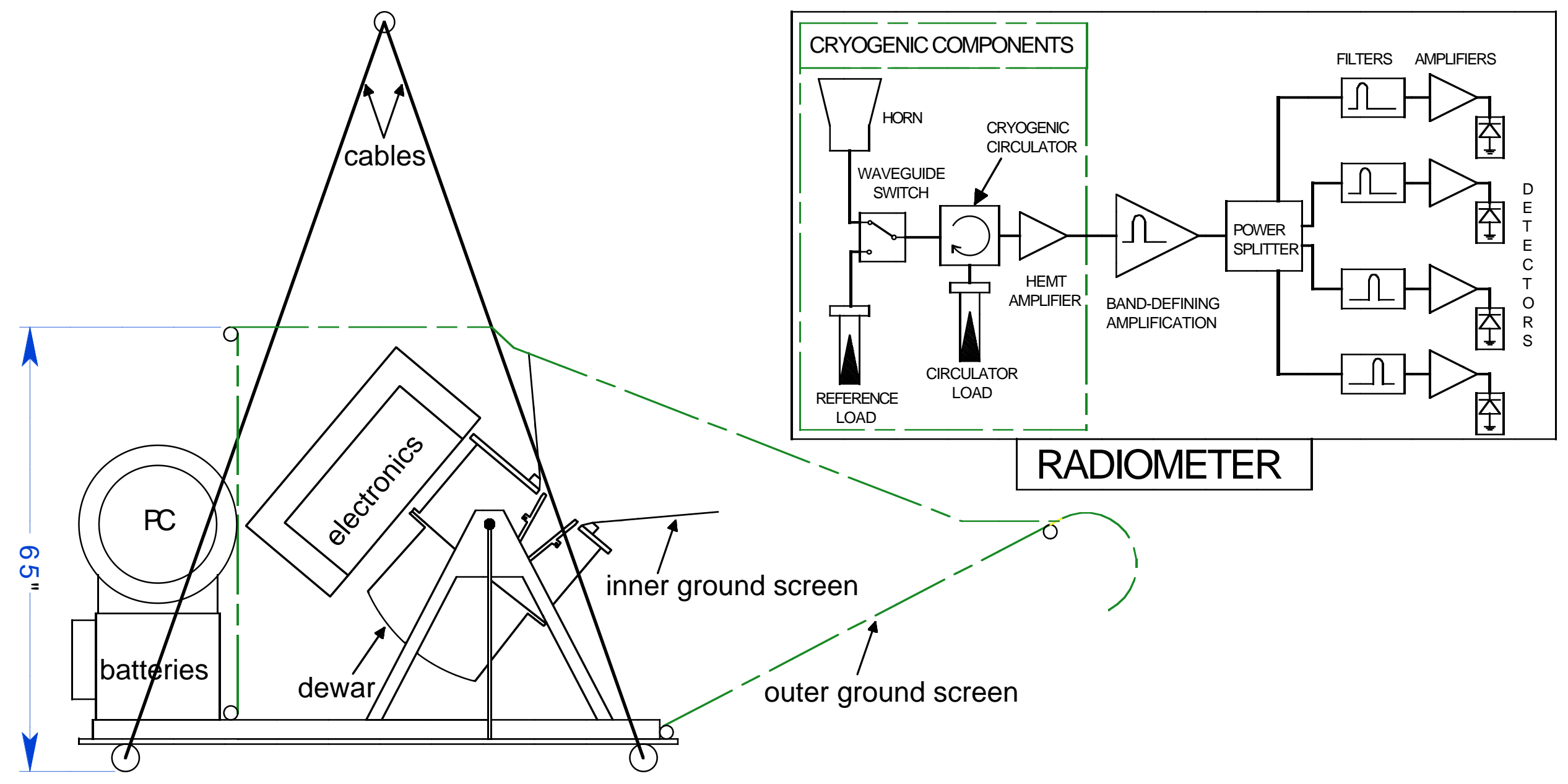

